\section{Malformations of Cerebral Cortical Development in Oral- Facial-Digital Syndrome Type VI}

Oral-facial-digital syndrome type VI (OFDS VI) or Váradi-Papp syndrome is a rare autosomal recessive disorder, distinguished from other type of OFDS by metacarpal abnormalities with central polydactyly; cerebellar abnormalities; and, sometimes, hypothalamic hamartomas. Affected patients present with hypotonia, cognitive impairment, irregular breathing, and oculomotor apraxia. The clinical presentation, combined with brain MR imaging features of the molar tooth sign, have resulted in OFDS VI being classified as one of the Joubert syndrome-related disorders. ${ }^{1,2}$ We report an additional 2 patients with OFDS VI whose MR imaging studies revealed malformations of cerebral cortical development as well as the molar tooth sign and a hypothalamic hamartoma.

Patient 1, a 13-year-old girl, was referred to our hospital because of psychomotor retardation ( $\mathrm{IQ}=46$ ), cerebellar ataxia, and horizontal nystagmus. She had bilateral polydactyly involving both feet and lingual nodules, which were surgically resected at age 1 year. Results on MR imaging revealed subependymal and subcortical heterotopia as well as the molar tooth sign and a hypothalamic hamar- toma (Fig $1 A, B)$. No renal or ocular anomalies were recognized on examination. Serum electrolytes and chemistries were normal, including those reflecting hepatic function. The clinical and radiologic features led to a diagnosis of OFDS VI. She presents no epilepsy or precocious puberty.

Patient 2 is a young girl who presented with polydactyly and partial syndactyly on both hands and feet, lingual nodules, and neonatal tachypnea and apnea that resolved spontaneously. She later manifested psychomotor retardation and hypotonia; epilepsy commenced at age 8 years. Results of a renal sonographic examination, ophthalmologic examination, and blood electrolytes/chemistries were normal. Results on MR imaging at age 8 years revealed a molar tooth sign, hypothalamic hamartoma, subependymal nodular heterotopia, and local polymicrogyria (Fig 2A,B). Combined clinical and radiologic features established a diagnosis of OFDS VI.

Results on MR imaging in the 2 patients with OFDS VI demonstrated malformations of cortical development, including heterotopia or polymicrogyria, in addition to molar tooth sign and hypothalamic hamartomas. Malformations of cortical development seem to be uncommon in OFDS VI (we were unable to find a previous report);
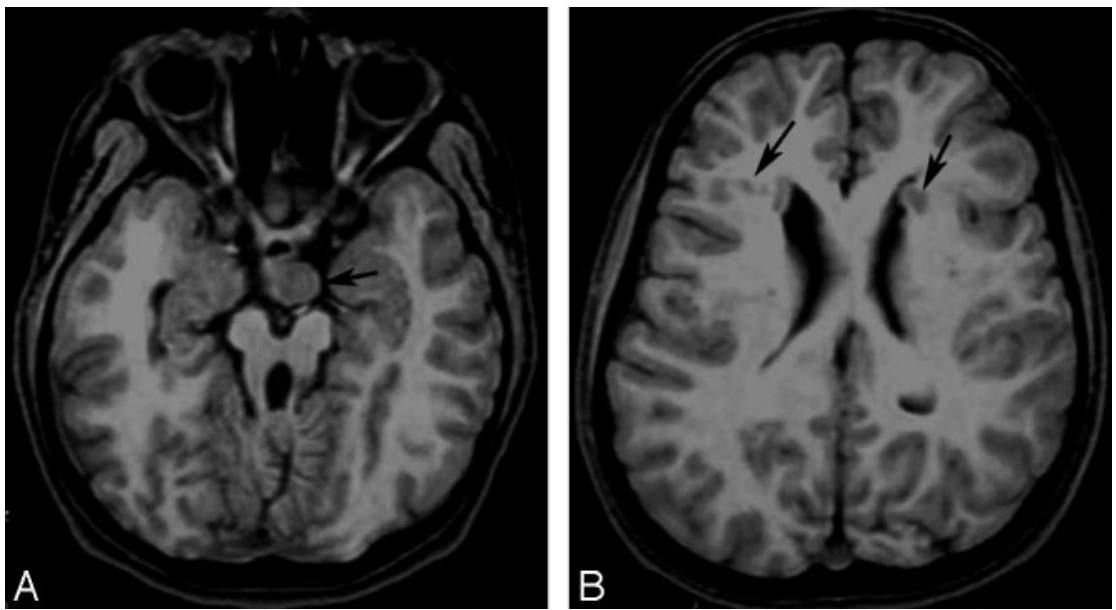

Fig 1. Patient 1, a 13-year-old girl. A, Axial echo-spoiled gradient-echo (TR, $50 \mathrm{msec}$; $\mathrm{TE}, 6 \mathrm{msec}$ ) images show the molar tooth sign of the midbrain and hypothalamic hamartoma (black arrow). B, Axial echo-spoiled gradient-echo (TR, $50 \mathrm{msec}$; TE, $6 \mathrm{msec}$ ) at the level of the bodies of the lateral ventricles shows subependymal (left) and subcortical (right) heterotopia (arrows)
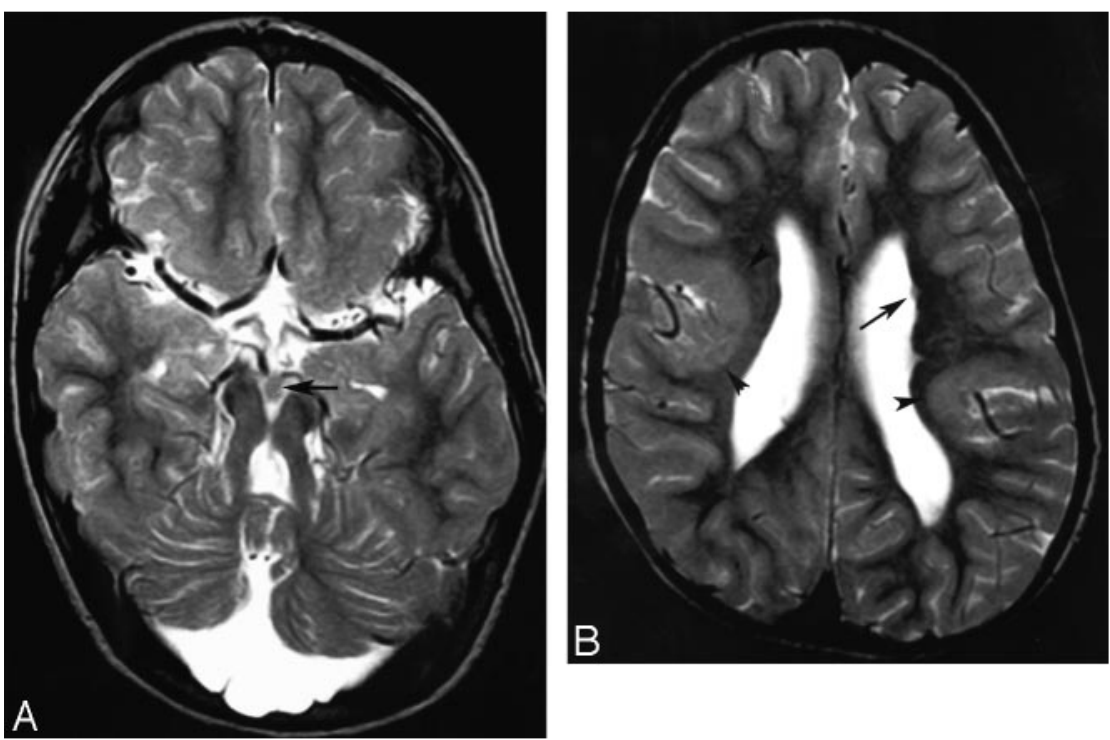

Fig 2. Patient 2, a 9-year-old girl. A, Axial T2-weighted image shows a molar tooth sign of the midbrain; small hamartoma (arrow); and a small, dysmorphic vermis. B, Axial T2-weighted image at the level of the bodies of the lateral ventricles shows subependymal nodular heterotopia (arrow) and bilateral foci of polymicrogyria (arrowheads). 
however, polymicrogyria is not uncommonly associated with the molar tooth sign in Joubert syndrome and related disorders. ${ }^{2}$ The finding of heterotopia or polymicrogyria in patients with OFDS VI suggests that an unknown factor may result in abnormal late cerebral migration or cortical organization, development of the molar tooth sign (midbrain-hindbrain disorder), and oral-facial-digital disorders.

\section{References}

1. Poretti A, Brehmer U, Scheer I, et al. Prenatal and neonatal MR imaging findings in oral-facial-digital syndrome type VI. AJNR Am J Neuroradio 2008;29:1090-91

2. Zaki MS, Abdel-Aleem A, Abdel-Salam GM, et al. Molar tooth sign: a new Joubert syndrome and related cerebellar disorders classification tested in Egyptian families. Neurology 2008;70:556-65

J. Takanashi

H. Tada

Department of Pediatrics

Kameda Medical Center

Kamogawa, Japan

H. Ozaki

Department of Pediatrics

Hiratsuka Kyosai Hospital

Hiratsuka, Japan

A. J. Barkovich

Department of Radiology

University of California-San Francisco

San Francisco, Calif

DOI 10.3174/ajnr.A1287 